\title{
Incidencia de la cultura patriarcal en la violencia contra la mujer y el desarrollo humano en los comités del vaso de leche del distrito de Concepción
}

Impact of patriarchal culture on violence against women and human development in the glass of milk committees of the Concepción district

Dora Gómez Ferrerl

Cintya Patricia Sánchez Chumpitasi2

RECIBIDO: 9 DE ABRIL DE 2020

ACEPTADO: 5 DE JUNIO DE 2020

\section{RESUMEN}

La violencia contra la mujer constituye en la agenda nacional el principal problema de las mujeres, la región Junín ocupa el tercer lugar a nivel nacional en violencia conyugal. En el distrito de Concepción la violencia contra la mujer es un problema álgido en la medida que tiene sectores vulnerables en su población, mujeres en abandono, madres solteras, mujeres analfabetas, migrantes, mujeres con bajos niveles de instrucción, entre otros. La mayor parte de esta población ha sido socializada en una cultura autoritaria y violenta sin embargo debido a que

\footnotetext{
' Licenciada en Sociología, Docente, Universidad Nacional del Centro del Perú, Perú; email: dgomez@uncp, ORCID: https://orcid.org/0000-0002-0427-6026

2 Licenciada en Sociología, Universidad Nacional del Centro del Perú, Perú; patriciasanchezch@gmail.com; ORCID: https://orcid.org/0000-0003-1056-0514
} 


\section{2}

permanentemente las mujeres de los comités del vaso de leche reciben capacitaciones acerca de sus derechos y contra la violencia de genero de parte del Ministerio de la Mujer y Poblaciones Vulnerables, expresan opiniones que cuestionan el machismo. El objetivo de esta investigación fue conocer la influencia de la cultura patriarcal en la generación de la violencia y el desmedro del desarrollo humano integral de estas mujeres, para lo cual se empleó el método cuantitativo, siendo una investigación de tipo descriptivo - explicativo se aplicó la escala Likert. El resultado obtenido nos demuestra que el 15\% de mujeres manifiesta influencia de la cultura patriarcal que se expresa en la violencia psicológica y económica que sufren obteniendo un desarrollo humano disminuido en comparación con el porcentaje de mujeres que no es víctima de violencia. La escala de Likert aplicada refleja las opiniones de las mujeres respecto a la cultura patriarcal, la violencia de género, la autoestima y empoderamiento de las mismas.

Palabras clave: violencia contra la mujer, cultura patriarcal, desarrollo humano, Concepción

\section{ABSTRACT}

Violence against women is the main problem for women on the national agenda, the Junín region ranks third nationally in marital violence. In the Concepción district, violence against women is a critical problem inasmuch as it has vulnerable sectors of its population, abandoned women, single mothers, illiterate women, migrants, women with low levels of education, among others. The majority of this population has been socialized in an authoritarian and violent culture, however, since the women of the glass of milk committees are permanently trained on their rights and against gender violence by the Ministry of Women and Vulnerable popul The objective of this investigation was to know the influence of the patriarchal culture in the generation of violence and the detriment of the integral human development of these women, for which the quantitative method was used, being a descriptive-explanatory investigation the Likert scale. The obtained result shows us that $15 \%$ of women manifest influence of the patriarchal culture that is expressed in the psychological and economic violence they suffer, obtaining a diminished human development in comparison with the percentage of women who are not victims of violence. The Likert 
scale applied reflects the opinions of women regarding patriarchal culture, gender violence, self-esteem and their empowerment.

Keywords: violence against women, patriarchal culture, human development, Concepción

\section{Introducción}

La violencia hacia la mujer es una problemática que afecta a los diversos estratos de la población femenina, las políticas sociales de género en violencia no han logrado bajar las cifras de violencia ni de feminicidio. Una de las mayores causas para la violencia es la cultura patriarcal que se ha instalado en nuestro país, la misma que se reproduce en muchos hogares y contra la cual se han venido haciendo pocos esfuerzos desde el estado. Es así que esta investigación tiene como objetivo principal conocer la opinión de las mujeres de los comités del vaso de leche acerca de la influencia de la cultura patriarcal en la violencia y el desarrollo humano integral. Para esto se tiene como hipótesis general que la cultura patriarcal influye directamente en la generación de la violencia contra la mujer y negativamente en el desarrollo humano de la mujer en el distrito de Concepción de la provincia de Concepción. La investigación pretende profundizar acerca de las raíces estructurales y de orden cultural que permita explicar la presencia cada vez más acentuada de este fenómeno. Es necesario teorizar acerca del sistema de género imperante, la cultura patriarcal y los discursos hegemónicos respecto a las identidades de género y el relacionamiento entre hombres y mujeres. Así mismo es necesario indagar acerca de los enfoques de género y de la violencia que acompañan la formulación de las políticas públicas respecto a la implementación de esta y el rol que juegan los funcionarios y autoridades locales y operadores del estado respecto a estas. La violencia contra la mujer tiene una incidencia negativa en la calidad de vida y en el desarrollo humano de las víctimas.

El objetivo formulado ha sido: Conocer la opinión de las mujeres en los comités del vaso de leche del distrito de Concepción en la provincia de Concepción acerca de la influencia de la cultura patriarcal en la violencia y el desarrollo humano integral 


\section{4}

Como hipótesis central se sustenta: La cultura patriarcal influye directamente en la generación de diversos tipos de violencia contra la mujer como son: la Psicológica, física, sexual y económica. Y en diversas dimensiones.

La influencia de la cultura patriarcal influye negativamente en el desarrollo humano de la mujer en el distrito de Concepción de la provincia de Concepción.

La presente investigación se justifica en la medida que la cultura patriarcal tiene una directa incidencia en la afectación en el desarrollo de las mujeres y el sometimiento de ella en los diversos tipos de violencia.

Las mujeres de los comités del vaso de leche y en particular en Concepción no logran percibir la cultura patriarcal como un problema estructural en la medida que han sido socializadas mediante la familia en un largo proceso de aprendizaje y naturalización del mismo. En este sentido encontramos coincidencias con lo planteado por Barros (2005) en su tesis doctoral Discriminación sexista y otras formas de violencia estructural e institucional contra la mujer" sostiene que identidad masculina, así como la femenina se configuran dentro de una estructura patriarcal que oprime y subordina a la mujer mediante la violencia. La violencia estructural e institucional está configurada a su vez por el sistema de género imperante.

Botello (2006) en su investigación establece la relación directa entre las categorías identidad, masculinidad y violencia. La pregunta de investigación fue: ¿̇Cuál es la relación entre violencia, identidad y género en hombres jóvenes mexicanos en un contexto determinado?.. Se llevaron a cabo 10 grupos de discusión con jóvenes varones en diferentes zonas de México. Se realizó análisis del discurso y se desarrollaron diferentes categorías que permitió ordenar lo analizado. La investigación es de carácter cualitativo y se trabajó bajo la técnica de grupo focal. La autora percibe que la masculinidad machista y patriarcal se va enfrentando a nuevas pautas y comportamientos que va imprimiendo la sociedad moderna.

Toldos (2004) El objetivo general de esta investigación es avanzar en la comprensión de cómo es el procesamiento de la información de los y las adolescentes ante situaciones de violencia hipotéticas en función del sexo y del estatus del agresor y de la víctima, además, se examina la relación entre dicho proceso y las actitudes hacia el género y la violencia, las creencias hacia grupos minoritarios, el uso de la violencia y los estereotipos de género de los y las adolescentes. 
Leal (2006) estructura su trabajo de investigación en dos partes: la primera trata de definir el concepto de "desarrollo integral de los países" para lo cual escoge los indicadores adecuados. Considera el desarrollo humano integral en varias dimensiones y campos como la evolución individual y colectiva del ser, la interrelación hombre-sociedad; de la economía, la cultura, vehículo básico de desarrollo; el desarrollo generalmente admitido por la comunidad internacional. En la segunda se toma en considera niveles de desarrollo humano según el criterio de naciones unidas (índice de desarrollo humano), se toma en consideración 72 países. Lujan (2013) El trabajo de investigación tiene como propósito considerar la violencia contra la mujer como una grave vulneración de los derechos humanos que afecta su salud física y psicológica que impide desarrollar plenamente sus capacidades y habilidades. La autora manifiesta que este tipo de violencia se presenta en la sociedad independientemente del estrato social, edad, cultura, religión, ámbito geográfico. El enfoque de esta problemática debe ser multidisciplinar. La autora sostiene que no bastan las normas legales, para erradicar este tipo de violencia se requieren cambios estructurales profundos bajo la concepción de equidad e igualdad de género. El trabajo aborda las distintas formas de violencia contra la mujer con la prostitución forzada, violencia física, psicológica, sexual, entre otras, así como los feminicidios. Así mismo la violencia en periodos de paz y de guerra.

Galego (2013) en su Tesis doctoral Autonomía personal y afrontamiento en mujeres en situación de maltrato aborda el citando lo siguiente: La exposición a la violencia de género provoca serios problemas de salud relacionados con cambios psicológicos vinculados al estrés (Dutton, 1992) que requieren activar procesos cognitivos y conductuales para su afrontamiento. La OMS señala que las mujeres en situación de maltrato a menudo tienen limitaciones en su comportamiento autónomo e independiente (WHO, 2010).

La autonomía constituye un factor fundamental para el desarrollo humano integral implica varias dimensiones entre las que cabe mencionar el autocontrol, la capacidad de desarrollo de estrategias para enfrentar problemas, así como el tomar decisiones independientes de presiones o chantajes emocionales. Para el desarrollo 


\section{6}

de la autonomía la mujer debe independizarse de la dependencia emocional y económica del varón.

Lizana (2014) en su tesis doctoral. Las mujeres y el poder colectivo. Análisis crítico del enfoque de empoderamiento de mujeres y la acción colectiva feminista en Chile. La autora señala que el empoderamiento se refiere a la transformación de las relaciones de poder opresivas entre los géneros. El objetivo de esta tesis fue realizar un análisis teórico y práctico, examinando, por un lado, los argumentos centrales respecto del aporte de la noción de justicia social y política de Iris Marion Young y las ideas sobre una ciudadanía más inclusiva desde el pensamiento feminista hacia el enfoque de empoderamiento de mujeres y, por otro lado, las visiones sobre el poder y la adquisición del mismo en las acciones colectivas y prácticas comunitarias de grupos de mujeres, organizaciones y colectivas/os feministas chilenas/os. La hipótesis de trabajo utilizada fue que no toda propuesta de empoderamiento permite avanzar en el horizonte del autodesarrollo y la autodeterminación, conceptos claves de Iris Marion Young, ya que se requiere un marco de transformación social feminista para desplegar todas las potencialidades del poder colectivo, tanto a nivel teóricofilosófico como a nivel de intervención social fundada.

En la tesis se ha utilizado el enfoque cualitativo e interpretativo y la metodología correspondió a un estudio de casos, de tipo colectivo y abierto. En cuanto a los resultados, analizo las convergencias entre los aportes de Iris Marion Young al enfoque de empoderamiento de mujeres y los posicionamientos y prácticas de los nueve grupos estudiados, el vínculo entre empoderamiento y ciudadanía, las características del empoderamiento en las prácticas comunitarias de los grupos, para concluir afirmando que el empoderamiento puede constituir una herramienta adecuada siempre que cumpla determinadas características asociadas a una propuesta más amplia, dentro de un proyecto de transformación feminista con impactos a nivel micro y macro social.. 


\section{Método}

La investigación se realizó con el método cuantitativo, siendo de tipo descriptivo explicativo, ya que se buscó describir las percepciones acerca de la violencia en las mujeres en el distrito de Concepción de la provincia de Concepción. Incidiendo en la correlación entre la cultura patriarcal y la violencia psicológica, física, sexual y económica de las mujeres y su influencia negativa en el desarrollo humano.

Para la investigación se trabajó con las mujeres miembros del Programa de "Vaso de Leche", del distrito de Concepción, provincia de Concepción, mismas que según su padrón son 210. De este total a través de una prueba estadística el número de muestra se fijó en 67 mujeres, a quienes se les aplicó en instrumento de recolección de datos que consistió en una escala Likert. Así mismo se realizó la prueba de fiabilidad de la escala, a través del Alfa de Cronbach mismo que arrojó la aprobación de todo el instrumento con 0.76 el cual es un valor aceptable. Para la Escala 1 el valor de alfa de Cronbach fue de 0.54 es el cual es un valor regular de fiabilidad. Para la Escala 2 el valor de alfa de Cronbach fue de 0.85 que es un valor aceptable y para la Escala 3 el valor de alfa de Cronbach fue de 0.57 considerado un valor aceptable.

\section{Resultados}

La investigación tuvo como propósito conocer las opiniones de las mujeres de los comités del vaso de leche del distrito de Concepción respecto a la cultura patriarcal y los distintos tipos de violencia que se ejercen contra la mujer, y el desarrollo humano integral de las mujeres.

La cultura patriarcal es un conjunto de postulados que representa la superioridad jerárquica del varón frente a la mujer, implica las relaciones inequitativas y desiguales de género. Así como la subordinación de las mujeres frente a los hombres, convirtiéndose en la causa principal de la violencia contra la mujer. Esta cultura patriarcal lo que hace es generar una serie de estereotipos que a su vez al ser asumidos por las mujeres y hombres convierten en naturales ciertas prácticas y normalizan expresiones de violencia. La escala para medir la Cultura Patriarcal en las mujeres entrevistadas arrojó el siguiente resultado: 


\section{8}

\section{Figura 1}

Escala para medir la Cultura Patriarcal

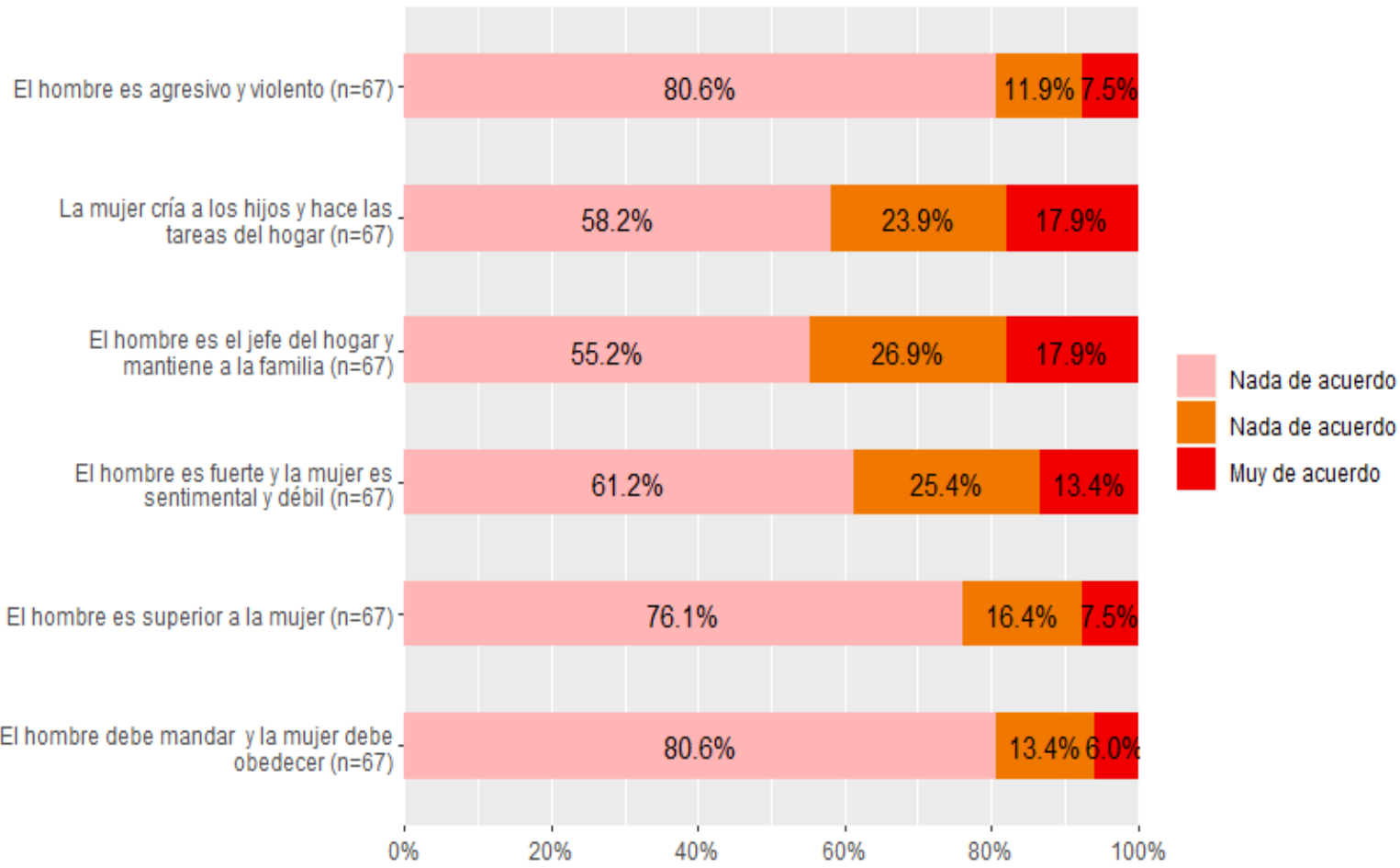

De En el gráfico se puede encontrar que si bien el $80.6 \%$ de las encuestadas no está de acuerdo en que la naturaleza del hombre sea violenta, pero hay un $7.5 \%$ que piensa que la agresividad y violencia son características propias de los hombres.

Se puede ver que un 58.2\% de las encuestadas no están de acuerdo con que la mujer sea quien tenga la exclusividad de las labores de crianza de los hijos y cuidado del hogar, esto se contrapone con el $17.9 \%$ de encuestadas que si considera que las mujeres son las que realizan estas labores. Esta perspectiva se corresponde con el $55.2 \%$ de mujeres no está de acuerdo con que el hombre sea el jefe del hogar y quien mantenga a la familia, siendo nuevamente el $17.9 \%$ de mujeres las que piensan que la manutención económica de la familia es deber del hombre.

El $61.2 \%$ de las encuestadas no está de acuerdo con las características patriarcales dadas hacia el hombre como fuerte y la mujer como sentimental y débil. Sin embargo, el $13.4 \%$ está de acuerdo. En la misma línea se tiene que el $76.1 \%$ no 
considera correcto que el hombre sea superior a la mujer, teniéndose un $7.5 \%$ que cree lo contrario.

Por último, se tiene que hay un $80.6 \%$ de mujeres encuestadas que no cree que el hombre deba mandar y la mujer obedecer, pero se encuentra un $6 \%$ que si está de acuerdo con ello.

La escala aplicada mide la opinión de las mujeres entrevistadas respecto a la cultura patriarcal la cual no refleja necesariamente sus experiencias ni relaciones de pareja. Se consideró dentro de la investigación una escala para medir la violencia contra la mujer, esta se subdividió en sub-escalas para la violencia psicológica y otra para medir la violencia económica. Es importante ver que en el caso de ambas escalas se muestra que hay un $10 \%$ que sufre ambos tipos de violencia, donde se muestra una correlación entre la violencia psicológica y la económica.

\section{Figura 2}

Escala para medir la Violencia Psicológica

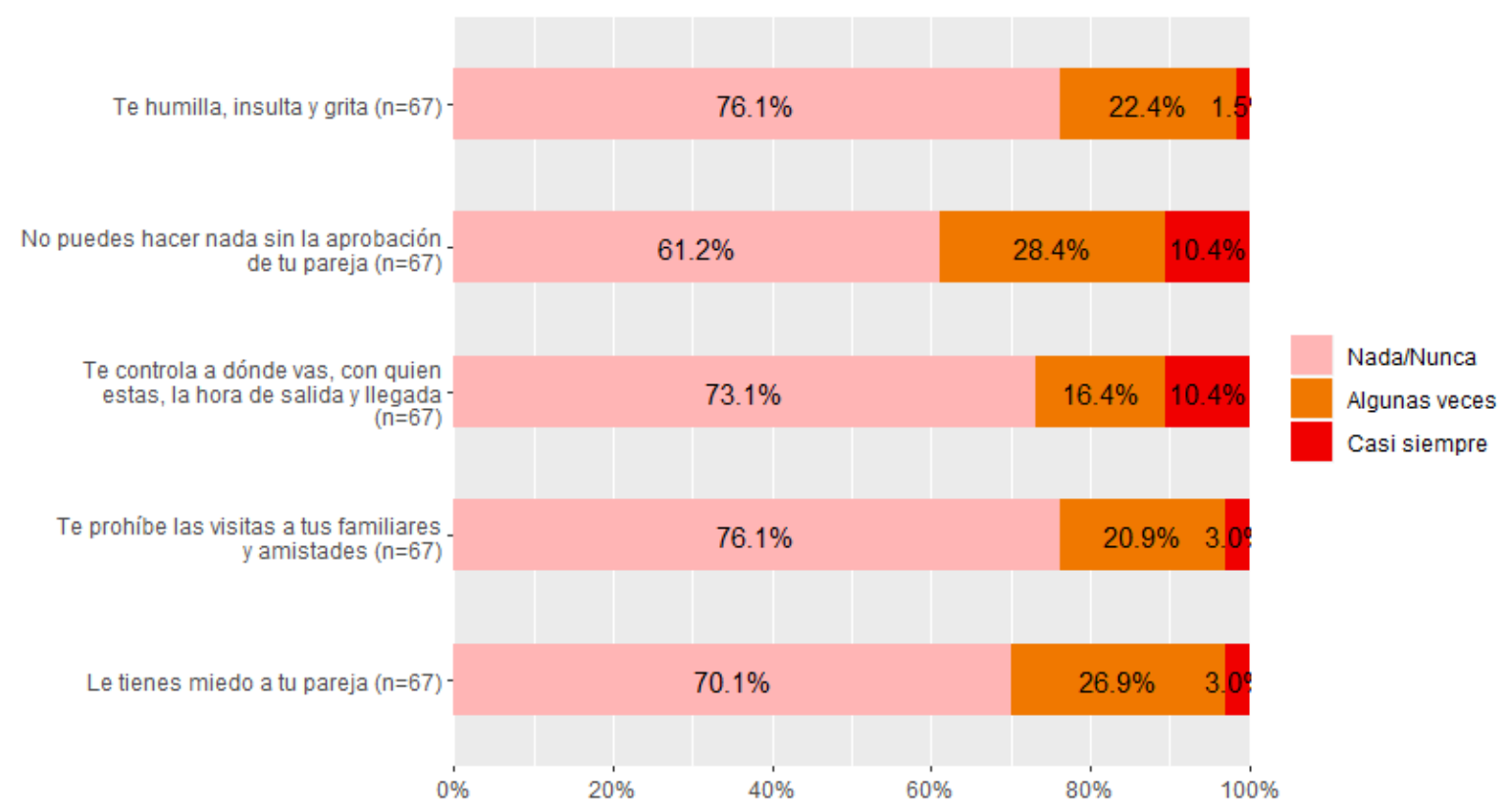

Para esta sub-escala se ha tomado en consideración lo relacionado a la violencia psicológica, se tienen que $76.1 \%$ de mujeres, es decir más del $70 \%$ de las encuestadas, manifiesta no haber sufrido ni insultos, humillaciones o gritos por parte 


\section{0}

de su pareja, se encuentra sin embargo que el resto de encuestadas al menos en una ocasión si ha recibido esta violencia.

El 61.2\% de encuestadas dice que puede realizar actividades sin la necesidad de pedir aprobación a su pareja, mientras que hay un $10.4 \%$ de mujeres que afirma no poder hacer ninguna actividad sin antes recibir la aprobación de su pareja. En la misma línea se tiene que el $73.1 \%$ de las mujeres encuestadas refiere que sus parejas no ejercen control sobre sus actividades personales, repitiéndose el $10.4 \%$ que manifiesta que su pareja si ejerce control.

Por otro lado, tenemos, que el $76.4 \%$ de las encuestadas afirman que sus parejas no les prohíben amistades y tampoco la visita de sus familiares, pero el resto de las encuestadas manifiesta que al menos una vez sus parejas les han prohibido una amistad o interacción con algún familiar.

Por último, se tiene que el $70.1 \%$ de las mujeres dicen no sentir temor de su pareja, el $29.9 \%$ como contraparte dicen que al menos en una ocasión ha sentido temor de su pareja.

De igual manera que en la escala anterior las mujeres entrevistadas refieren opiniones que deben constituir ideales en la convivencia y relación entre la pareja basada fundamentalmente en las charlas y talleres que acerca de la violencia y derechos de mujer reciben de parte del Centro de Emergencia Mujer de Concepción. 


\section{Figura 3}

Escala para medir la Violencia Económica

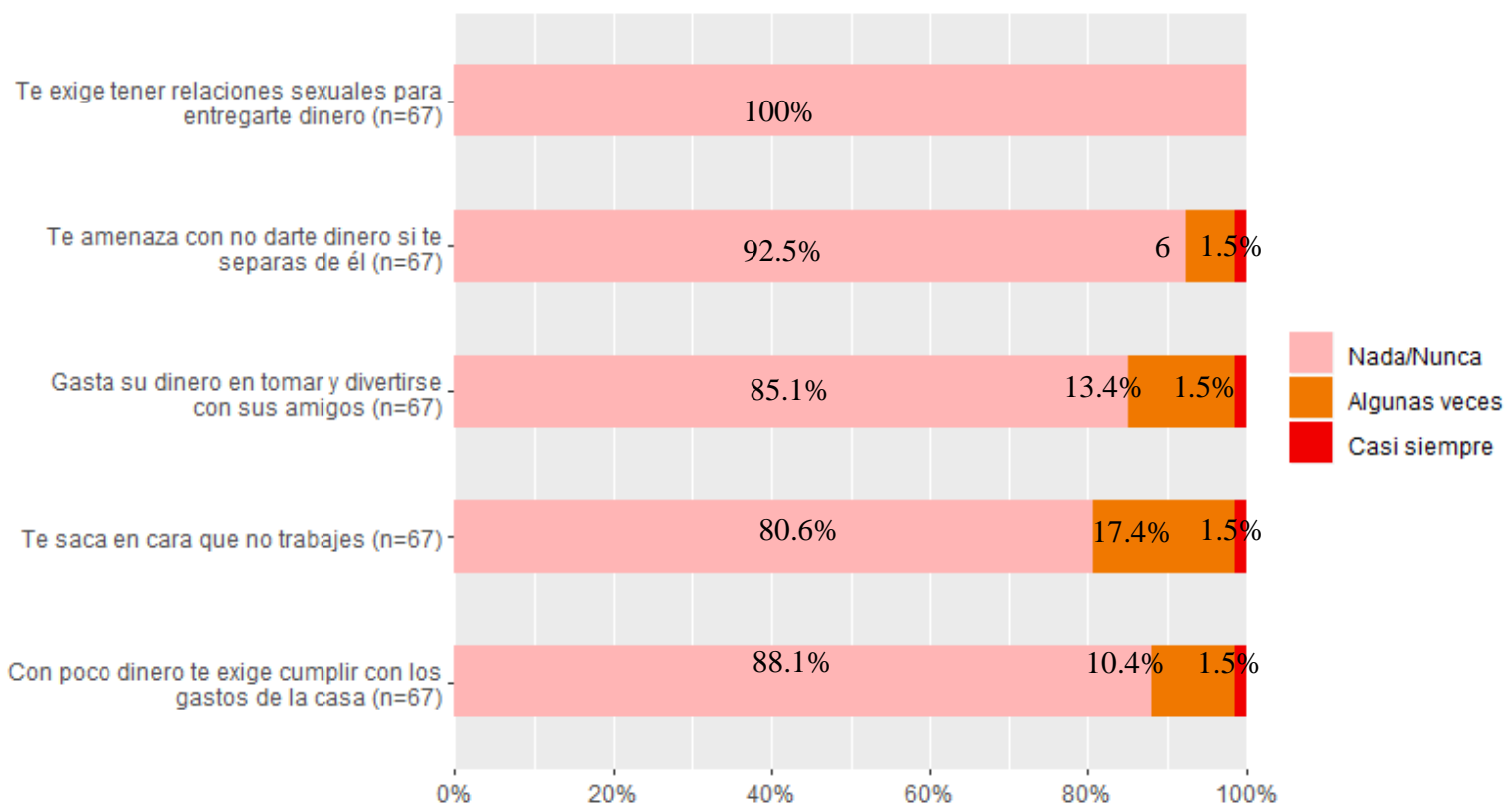

Esta sub-escala nos revela que ninguna de las encuestadas ha sufrido un tipo de acoso sexual para el acceso a dinero. También se tiene que el $92.5 \%$ de las encuestadas dice nunca ha sufrido ningún tipo de chantaje económico, ante la posibilidad de separarse. Sin embargo, hay un $7 \%$ de encuestadas que ha manifestado haber sido amenazada con el retiro de dinero si es que hay una separación.

Por otro lado, el $85.1 \%$ dice que sus parejas no hacen uso de todo su dinero en bebidas alcohólicas o salidas con sus amigos, habiendo $14.9 \%$ de mujeres encuestadas que dicen que al menos una vez sus parejas si han gastado su dinero en alcohol.

El $80.6 \%$ de encuestadas dice que sus parejas nunca les ha reclamado que sean ellos los que mantengan el hogar, mientras que el resto de encuestadas si ha vivido esta situación al menos una vez.

Se encontró que el $11.9 \%$ de encuestadas dice que en al menos en una ocasión sus parejas les han exigido cumplir con todos los gastos de la casa con poco dinero, situación que el $88.1 \%$ no ha pasado. 
Incidencia de la cultura patriarcal en la violencia contra la mujer y el desarrollo humano en los comités del vaso de leche del distrito de Concepción

\section{2}

Las respuestas de las entrevistadas reflejan percepciones ideales como no debe darse y evitarse la violencia económica.

Tenemos también una escala para medir el desarrollo humano integral, donde se resalta dos variables autoestima y empoderamiento de la mujer.

\section{Figura 4}

Escala para medir la Autoestima

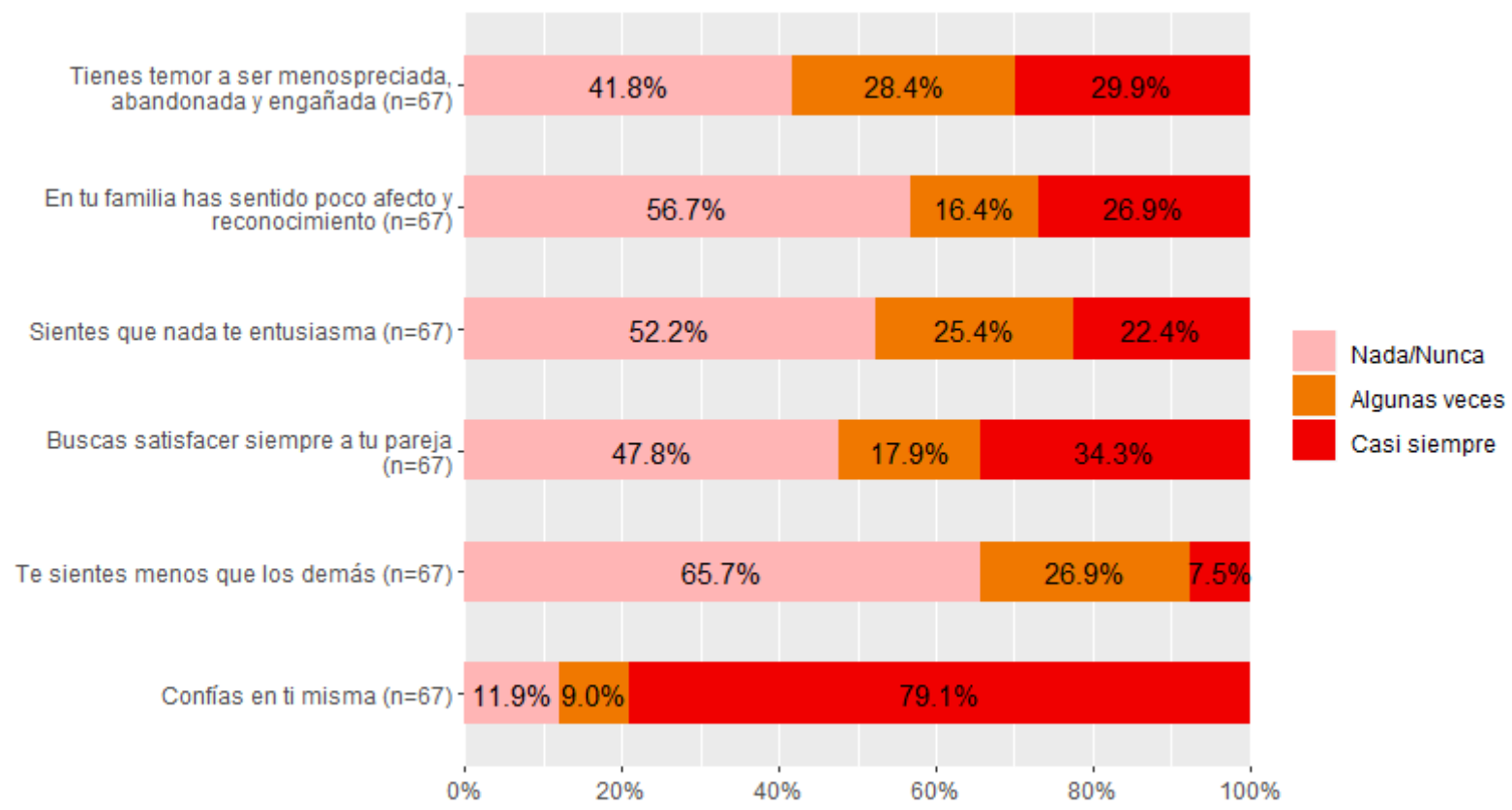

Para el caso de la autoestima se pusieron varios ítems que las mujeres encuestadas pudieran identificar con facilidad, es así que tenemos que el $41.8 \%$ no tiene temor de ser menospreciada, engañada o abandonada, mientras que el $29.9 \%$ tienen este temor como una constante.

Encontramos que el $56.7 \%$ de mujeres no se siente que haya habido poco afecto 0 reconocimiento en sus familias. Sin embargo, hay un $26.9 \%$ que si ha sentido que en su familia no había afecto hacia ella. 
Hay un $22.4 \%$ de mujeres que afirma que no encuentra entusiasmo en ninguna actividad, existe un $25.4 \%$ que en ocasiones no tienen entusiasmo para desarrollar sus actividades y un $52.2 \%$ que tiene un entusiasmo constante.

El $65.7 \%$ de encuestadas que siente bien consigo misma, pero se encuentra que el 7.5\% siempre se siente menos que las demás personas.

Por último, se tiene que el $11.9 \%$ de mujeres no confía en sí misma, habiendo un $79,1 \%$ que afirma que tiene confianza en ellas mismas.

En las respuestas a la escala en la dimensión de la autoestima es necesario resaltare la categoría discriminación un porcentaje aproximado del $28 \%$ de mujeres señala haberse sentido menos que los demás y menospreciada. En nuestro país existen fuertes desigualdades sociales y discriminación por el racismo, la cultura patriarcal, las brechas sociales y económicas, el deficiente ejercicio ciudadano entre otros.

\section{Figura 5}

Escala para medir el empoderamiento

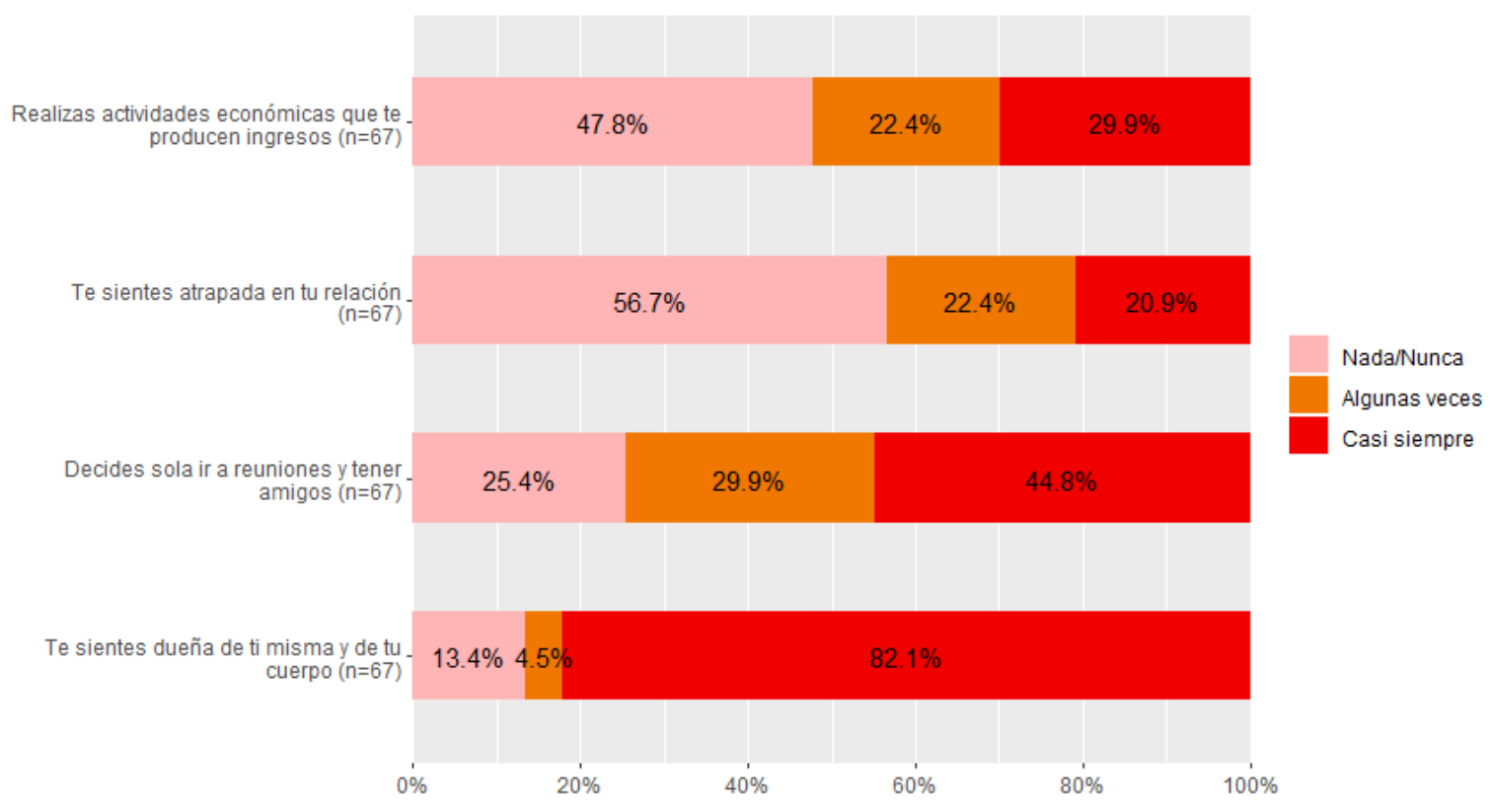

De acuerdo a este grafico el $\mathbf{2 9 . 9 \%}$ de las mujeres cuentan con ingresos lo cual significa un proceso hacia el empoderamiento, se considera un proceso en la medida que es importante el hecho de ir enfrentando la dependencia económica. 


\section{4}

Sin embargo, se observa que un $47.8 \%$, es decir casi un $50 \%$, de las entrevistadas que no reportan actividades que generen ingresos, lo cual constituye un obstáculo para tomar decisiones sobre proyectos de vida.

Un elemento positivo a destacar que un $56.7 \%$ no se siente atrapada en su relación. Sin embargo, un $20.9 \%$ estará contestando en un sentido contrario.

La autonomía en relación a las respuestas en las que las mujeres entrevistadas es muy débil, en la medida que cerca del 50\% estaría decidiendo acerca de su relacionamiento con amistades.

Un dato que es importante, es que el $13.4 \%$ no se siente dueña de sí misma ni de su cuerpo, lo que demuestra que hay un nivel de sumisión en estas mujeres.

La escala número 5 referida al empoderamiento estaría reflejando que todavía las mujeres tienen dependencia económica de la pareja (47.8\%) realidad que constituye un obstáculo para la toma decisiones en lo que respecta a su crecimiento y desarrollo personal.

Los Comités de Vaso de Leche de Concepción ante la falta de otras organizaciones de base de mujeres, reciben capacitaciones constantes de parte del Centro de Emergencia Mujer, por lo cual al menos teóricamente conocen sus derechos como mujeres. Esto se refleja en la escala de Likert que se ha aplicado, sin embargo, la violencia sigue siendo un asunto que aún no se ha resuelto en la experiencia familiar, en la escuela y relaciones de pareja establecidas. Lo que refleja la escala son percepciones y no necesariamente parte de su realidad cotidiana.

\section{Discusión}

En esta parte se procederá al análisis, comparación y discernimiento de los resultados expuestos en diferentes investigaciones con los hallados en el presente trabajo. Las respuestas obtenidas en la dimensión de la escala referida al patriarcado reflejan en las mujeres de los comités del vaso de leche del distrito de Concepción conocimientos de identidad de género, masculinidad, violencia y cultura patriarcal en este sentido se encuentra una proximidad con la investigación de Botello (2006) que establece la relación directa entre las categorías en mención. Esta relación de variables se llevó a cabo en 10 grupos de discusión con jóvenes varones en diferentes zonas de México. La investigación es de carácter cualitativo y se trabajó bajo la 
técnica de grupo focal. En comparación la escala de Likert demuestra que las entrevistadas son mujeres de organizaciones de base que van desarrollando conocimientos y conciencia acerca de inequidades de género y la cultura patriarcal. En estas coincidencias podemos mencionar el trabajo de Toldos (2004) cuyo objetivo general está en la comprensión de cómo es el procesamiento de la información de los y las adolescentes ante situaciones de violencia hipotéticas en función del sexo y del estatus del agresor y de la víctima, además, se examina la relación entre dicho proceso y las actitudes hacia el género y la violencia, las creencias hacia grupos minoritarios, el uso de la violencia y los estereotipos de género de los y las adolescentes.

La investigación realizada refleja que las identidades de género y por tanto la violencia contra la mujer están configuradas desde la estructura cultural del patriarcado nos aproxima al enfoque desarrollado por Barros (2005) en su tesis doctoral Discriminación sexista y otras formas de violencia estructural e institucional contra la mujer" que sostiene que identidad masculina así como la femenina se configuran dentro de una estructura patriarcal que oprime y subordina a la mujer mediante la violencia. La violencia estructural e institucional está configurada a su vez por el sistema de género imperante.

En la medida que la investigación desarrollada pretende demostrar que la cultura patriarcal tiene incidencia directa en la violencia contra la mujer y negativa respecto al desarrollo humano integral de la mujer es pertinente referir el trabajo desarrollado por Leal (2006) pues considera el desarrollo humano integral en varias dimensiones y campos como la evolución individual y colectiva del ser, la interrelación hombresociedad; de la economía, la cultura, vehículo básico de desarrollo que viene a constituir el desarrollo concebido por la comunidad internacional.

Se ha considerado para abordar el desarrollo humano integral de la mujer de los comités del vaso de leche del distrito de Concepción dos variables básicas la autonomía y el empoderamiento. Las respuestas obtenidas respecto a la autonomía nos refieren que un $29.9 \%$ tiene temor a ser menospreciada engañada $y$ abandonada, $34.3 \%$ busca satisfacer siempre a su pareja, un $26.9 \%$ se siente menos que los demás. Esta información obtenida nos demuestra debilidades en la autoestima y autonomía de estas mujeres que constituye el primer peldaño en la 


\section{6}

toma de decisiones para el desarrollo humano integral. La falta de autonomía de la mujer se da en un contexto de dependencia emocional de la mujer que es una condición para el ejercicio de la violencia contra ella. En este sentido es necesario referir a Lujan que considera la violencia contra la mujer como una vulneración de los derechos humanos y un grave atentado contra su salud e integridad física y psicológica he impide el desarrollo de sus capacidades y habilidades. . La autora sostiene que no bastan las normas legales, para erradicar este tipo de violencia se requieren cambios estructurales profundos bajo la concepción de equidad e igualdad de género. Así mismo es necesario considerar el trabajo de Galego (2016) que en base a la definición de la Organización Mundial de la Salud señala que las mujeres en situación de maltrato a menudo tienen limitaciones en su comportamiento autónomo e independiente.

Para abordar la variable de empoderamiento de la mujer es útil la perspectiva desarrollada por Lizana en su trabajo de investigación. La autora señala que el empoderamiento se refiere a la transformación de las relaciones de poder opresivas entre los géneros, considera las acciones colectivas y prácticas comunitarias de grupos de mujeres, organizaciones sociales y colectivos feministas. Existe coincidencia en la perspectiva de la relación intrínseca entre empoderamiento y ciudadanía

El empoderamiento de mujer tiene como premisa la independencia emocional y económica respecto a la pareja, la autonomía y el desenvolvimiento en el espacio público. Constituye un proceso que permite desarrollar capacidades y habilidades personales en el ámbito de un proyecto colectivo. En las respuestas de la mujeres a quienes se les aplicó la escala de lickert encontramos que un $47.8 \%$ no realiza ninguna actividad económica que le genere ingresos. 


\section{Referencias}

Barros, L (2005) Discriminación sexista y otras formas de violencia estructural e institucional contra la mujer. Tesis doctoral. Universidad Carlos III de Madrid, España. Recuperado de http://hdl.handle.net/10016/519

Botello, L (2006) Identidad, masculinidad y violencia de género. Tesis doctoral. Universidad Complutense Madrid, España. Recuperado de https://eprints.ucm.es/7184/

Cantera, L (2004). Más allá del género. Nuevos enfoques de nuevas dimensiones y direcciones de la violencia en la pareja. Athenea Digital - num 6 Universidad Autónoma de Barcelona, España Recuperado de https://atheneadigital.net/article/view/n6-cantera/174-html-es

Galego, V (2013) Autonomía personal y afrontamiento en mujeres en situación de maltrato. Tesis doctoral. Ministerio de Sanidad, Servicios Sociales e Igualdad Centro de Publicaciones. Mdrid, España. Recuperado de https://violenciagenero.igualdad.gob.es/violenciaEnCifras/estudios/Tesis/pdfs /Tesis2_Autonomia_Personal.pdf

Hernández, R. Fernandez, C, Baptista, MP (2014). Metodología de la Investigación, 60 edición. México: Mc Graw Hill. Recuperado de https://www.academia.edu/37071176/Metodolog\%C3\%ADa_de_la_inve stigaci\%C3\%B3n_6a._ed._

Leal (2007) El desarrollo integral de los países desde un análisis multivariante. Tesis doctoral. Universidad Nacional de Educación a Distancia, Madrid, España

Lizana, $N$ (2014). Las mujeres y el poder colectivo. Análisis crítico del enfoque de empoderamiento de mujeres y la acción colectiva feminista en Chile. Universidad de Barcelona, España Tesis Doctoral. Recuperado de: https://dialnet.unirioja.es/servlet/tesis? codigo=93138

Lujan, P (2013) La violencia contra las mujeres y alguien más... Tesis doctoral. Universidad de Valencia, España. Recuperado de: https://dialnet.unirioja.es/servlet/tesis? codigo $=80814$

Ortiz, A (2006) "Violencia doméstica modelo multidimensional y programa de intervención". Tesis doctoral. Universidad Complutense de Madrid, España. Recuperado de https://eprints.ucm.es/7199/ 
Incidencia de la cultura patriarcal en la violencia contra la mujer y el desarrollo humano en los comités del vaso de leche del distrito de Concepción

\section{8}

Toldos (2004) Adolescencia, Violencia y Género. Tesis doctoral. Universidad Complutense de Madrid, España. Recuperado de https://eprints.ucm.es/4477/1/T26325.pdf 